\title{
Productividad primaria del fitoplancton en la bahía de Mochima, Venezuela*
}

\author{
Federico González ${ }^{1}$, Evelyn Zoppi de Roa $^{1}$ y Edie Montiel $^{1}$ \\ ${ }^{1}$ Instituto de Zoología Tropical, Facultad de Ciencias, Universidad Central de Venezuela \\ Apt 47058 Caracas 1041, Venezuela
}

\begin{abstract}
RESUMEN. Se analizaron las fluctuaciones temporales y espaciales de los parámetros físicos, químicos, clorofila-a y productividad primaria durante periodos de surgencia y no surgencia en la bahía de Mochima, Venezuela. La columna de agua se dividió en cuatro estratos para determinar los parámetros físicos y químicos; $\mathrm{y}$ en dos estratos para determinar la clorofila-a y productividad primaria. La productividad primaria en no surgencia varió de 0 a $417 \mathrm{mg} \mathrm{C} \cdot \mathrm{m}^{-3} \cdot \mathrm{h}^{-1}$ y en surgencia de 76 a $426 \mathrm{mg} \mathrm{C} \cdot \mathrm{m}^{-3} \cdot \mathrm{h}^{-1}$. La concentración de clorofila-a en no surgencia fue de 1,11 a $8,57 \mathrm{mg}$ clor-a $\cdot \mathrm{m}^{-3} \mathrm{y}$ en surgencia de 4,21 a 44,59 mg clor-a $\cdot \mathrm{m}^{-3}$. La productividad primaria y la biomasa entre ambos periodos fue elevada, particularmente en surgencia. En ambos periodos los organismos fitoplanctónicos presentaron fuertes cambios temporales intradiarios que se manifestaron de acuerdo a eventos Lasker.
\end{abstract}

Palabras clave: fitoplancton, clorofila-a, productividad primaria, surgencia, bahía de Mochima, Venezuela.

\section{Primary productivity of phytoplankton in Mochima Bay, Venezuela*}

\begin{abstract}
The temporal and spatial fluctuations of physical and chemical parameters, chlorophyll-a, and primary productivity were analyzed during periods of upwelling and non-upwelling in Mochima Bay, Venezuela. The water column was divided into four strata for determining the physical and chemical parameters and into two strata for determining chlorophyll-a and primary productivity. Primary productivity varied from 0 to $417 \mathrm{mg} \mathrm{C} \cdot \mathrm{m}^{-3} \cdot \mathrm{h}^{-1}$ under non-upwelling conditions and from 76 to $426 \mathrm{mg} \mathrm{C} \cdot \mathrm{m}^{-3} \cdot \mathrm{h}^{-1}$ during upwelling. Chlorophyll-a concentrations ranged from 1.11 to 8.57 $\mathrm{mg}$ clor-a $\cdot \mathrm{m}^{-3}$ (non-upwelling) and from 4.21 to $44.59 \mathrm{mg}$ clor-a $\cdot \mathrm{m}^{-3}$ (upwelling). Primary productivity and biomass were elevated under both upwelling and non-upwelling conditions, particularly during upwelling. Sharp, temporal, daily changes were observed in phytoplanktonic organisms under both upwelling and non-upwelling conditions; these changes were manifested according to Lasker events.
\end{abstract}

Key words: primary productivity, phytoplankton, biomass, upwelling, Mochima Bay, Venezuela.

Autor corresponsal: Evelyn Zoppi de Roa (ezoppi@strix.cien.ucv.ve)

\section{INTRODUCCIÓN}

Los ecosistemas marinos son complejos y presentan jerarquías en su estructura y, de acuerdo al comportamiento del ambiente, se hace necesario estudiar las variaciones a pequeña escala ya que los trabajos a gran escala las ignoran (Mullin, 1993). Se considera que la alta frecuencia en la toma de muestras del plancton, en especial el fitoplancton, tiende a minimizar el error temporal asociado, el cual está calculado en un $35 \%$ en toma de muestras mensua- les en ecosistemas variables, según Moncoiffé et al. (2000).

Los eventos de surgencia han sido muy estudiados especialmente por Fortier \& Gagné (1990) y D'Croz et al. (1991), entre otros. Sin embargo, las referencias existentes según algunos autores (Lasker, 1975; Dickey, 1990; Osborn et al., 1990; Kiorboe, 1993; Montecinos \& Balbontín, 1993), se han basado principalmente en eventos hidrodinámicos, cambios de temperatura y densidad del agua.

* Trabajo presentado en el XXV Congreso de Ciencias del Mar de Chile y XI Congreso Latinoamericano de Ciencias del Mar (COLACMAR), realizados en Viña del Mar, entre el 16 y 20 de mayo de 2005. 
El transporte de aguas superficiales por el viento se basó por primera vez en la teoría de Ekman (1905) citado por Bakun (1973). Este transporte de masas de agua produce un sistema de corrientes en el mar abierto, provocado por el coeficiente de arrastre del viento sobre la superficie del mar. Las corrientes que se producen y el crecimiento del fitoplancton por el ascenso de los nutrientes son de vital importancia, ya que durante estos eventos, se produce el mayor reclutamiento de larvas de peces (Cury \& Roy, 1989). Las variaciones de estos procesos producen un enriquecimiento de la comunidad fitoplanctónica, que a corta escala de tiempo (días) incrementa la comunidad zooplanctónica. La variabilidad de este proceso se conoce como efecto Lasker y ha sido utilizado para explicar el éxito de las cohortes de peces bajo la acción intermitente del viento. La importancia de estos procesos se encuentra bajo alta necesidad de corroboración empírica. La bahía de Mochima es un cuerpo de agua importante por estar ubicado dentro del área de surgencia en la zona nororiental de Venezuela. En esta bahía existe una influencia oceánica relacionada con la topografía submarina, en la cual la acción de los vientos ejerce fuerza, ya que cuando éstos soplan del este con mayor intensidad (enero a abril), desplazan las masas de agua de las capas más superficiales. Cuando los vientos disminuyen progresivamente y cesan (octubre a diciembre) las masas de agua se estabilizan (Kato, 1961; Fukuoka, 1965, 1966; Okuda et al., 1968). Estos fenómenos de surgencia aportan gran cantidad de nutrientes a la superficie dando origen a un enorme crecimiento del fitoplancton (Kato, 1961).

La dinámica del plancton asociada a este fenómeno, permite pronosticar los cambios, basados en la velocidad del viento, los cuales demuestran que estos eventos son irregulares en el tiempo y producen cambios notables a pequeña escala en los diferentes eslabones de esta comunidad.

El presente trabajo tiene como objetivo analizar los cambios producidos por la velocidad del viento en los factores físicos y químicos, la productividad primaria y la concentración de clorofila-a ante eventos de surgencia y no surgencia en la bahía de Mochima.

\section{MATERIALES Y MÉTODOS}

La bahía de Mochima ubicada dentro del Parque Nacional Mochima, está situada en la vertiente norte de la cordillera de la costa $\left(64^{\circ} 19^{\prime} 30^{\prime \prime}-64^{\circ} 22^{\prime} 30^{\prime \prime} \mathrm{W}\right.$, $\left.10^{\circ} 24^{\prime}-10^{\circ} 20^{\prime} \mathrm{N}\right)$. La bahía está circundada por montañas en toda su extensión y abierta al norte por un canal de $1,7 \mathrm{~km}$ de ancho por $60 \mathrm{~m}$ de profundidad que se comunica directamente con la fosa de Cariaco, abarcando un área total estimada de $10,5 \times 10^{6} \mathrm{~m}^{2}$ y un volumen de $196 \times 10^{6} \mathrm{~m}^{3}$ haciendo frente a la influencia de los vientos alisios del noroeste (Okuda et al., 1968) (Fig. 1).

Para el estudio se hicieron dos salidas a terreno, una en época de no surgencia (noviembre de 1988, con valores mínimos en la velocidad del viento) y otra, en época de surgencia (febrero de 1999, con la mayor velocidad del viento). La toma de muestras se realizó durante 12 y 10 días cada una respectivamente, para comparar los periodos de viento fuerte y de calma, y evaluar los procesos a diferentes escalas. Se tomaron muestras al azar estratificadas y pareadas de los parámetros físicos y químicos, y de productividad primaria en la zona de la boca, entre 0 y $60 \mathrm{~m}$. Esta profundidad se toma como referencia, ya que en los casos estudiados sobre el efecto de Ekman, se ha estimado que alcanza profundidades de 50 a $100 \mathrm{~m}$ (Lasker, 1975; Kiorboe, 1993; Gómez, 1996).

La columna de agua de 0 a $60 \mathrm{~m}$ se dividió en cuatro estratos: Estrato 1: 0-10 m, Estrato 2: 10-20 $\mathrm{m}$, Estrato 3: 20-40 $\mathrm{m}$ y Estrato 4: 40-60 m. Los parámetros físicos y químicos se registraron hasta $60 \mathrm{~m}$ de profundidad, la clorofila y productividad primaria, solo en los estratos de 0-10 y 10-20 m hasta el punto de compensación de luz, calculado con el disco de Secchi.

Para la toma de muestras de clorofila y productividad primaria se utilizó una botella Van Dorn de 4 L. Para esta última, se utilizó el método de oxígeno de botellas claras y oscuras (Strickland, 1960; APHA, 1975). Para determinar la concentración de clorofila-a y feofitina, se utilizaron métodos espectrofotométricos (Strickland \& Parson, 1968; APHA, 1975). Para el análisis de la feofitina, se acidificaron las muestras con 0,02 $\mathrm{mL}$ de ácido clorhídrico según Margalef (1965) y APHA (1975).

Se determinaron las medias y las desviaciones estándar para eliminar las variables con sus desviaciones estándar más pequeñas (menores de 0,5) (Velilla, 1995). En cada uno de estos análisis se seleccionaron las variables más importantes utilizando el Análisis de Componentes Principales con uso de gráficos biplot. El criterio de selección de los componentes fue el de los valores propios menores de 1 (Chatfield \& Collins, 1980).

Se hicieron comparaciones de variables en dife- 
rentes condiciones. En el análisis de los resultados se utilizó el t de Student, para muestreos independiente y dependiente, con el fin de determinar diferencias interdiarias, entre estratos y entre periodos. Como variables independientes se utilizó profundidad, hora de la mañana y tarde. Como variables dependientes se consideró transparencia, temperatura, salinidad, clorofila-a, feofitina, productividad bruta, productividad neta y respiración.

Para su análisis se utilizaron los programas JMP versión 3.2.1 1997, MINITAB release 13.20.2000, STATA versión 7.0 2001 y SPAD versión 3.0 1999.

\section{RESULTADOS}

La velocidad del viento durante 1998 y 1999 mostró que el periodo con menores velocidades fue de julio a diciembre (no surgencia) y el de mayores de febrero a junio (surgencia) (Fig. 2).

Durante el periodo de no surgencia, las mayores temperaturas se registraron en superficie $\left(26,7^{\circ} \mathrm{C}\right)$, luego disminuyeron hasta $24,3^{\circ} \mathrm{C}$, formando una termoclina a partir de los $10 \mathrm{~m}$. Los menores valores se obtuvieron en los estratos más profundos, alcanzando un mínimo de $23,3^{\circ} \mathrm{C}$. En el periodo de surgencia la temperatura disminuyó, con respecto al periodo de calma, mostrando valores más o menos constantes en la columna de agua, con valores de 21,9 a $22,5^{\circ} \mathrm{C}$ (Fig. 3).

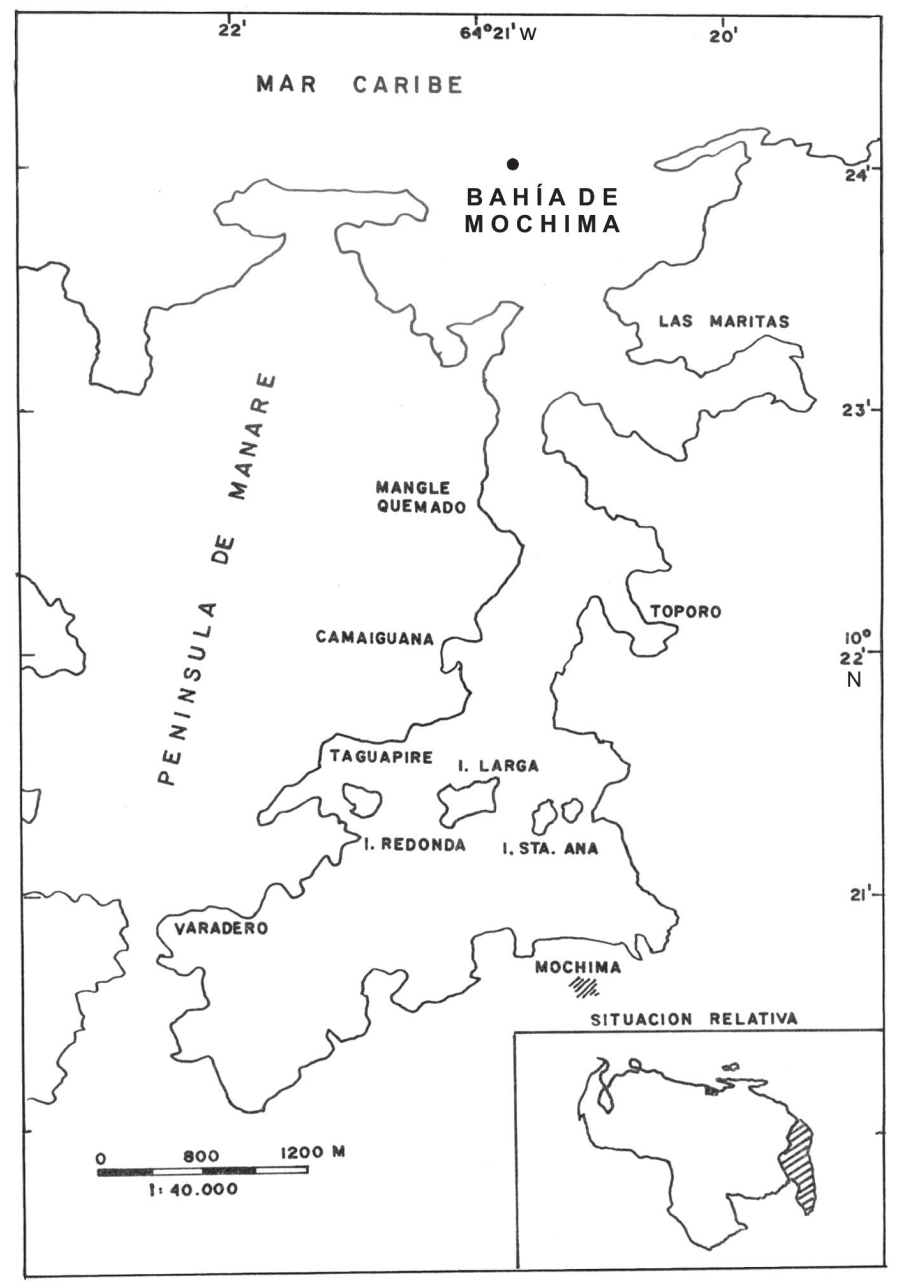

Figura 1. Localización de la estación de muestreo en la Bahía de Mochima.

Figure 1. Location of samplig station in in Mochima Bay. 
La concentración de oxígeno disuelto en no surgencia presentó fluctuación a lo largo del periodo, donde los valores del primer y segundo estrato, en general, tuvieron el mismo comportamiento $(\alpha \leq$ $0,05)$. De $0-10 \mathrm{~m}$ fue de $5,7-7,7 \mathrm{mg} \mathrm{O} \cdot \mathrm{L}^{-1}$ y a $10-20$ $\mathrm{m}$ fue de 5,16-8,6 $\mathrm{mg} \mathrm{O}_{2} \cdot \mathrm{L}^{-1}$ (Fig. 4a). En surgencia, igual que en no surgencia, la concentración de oxígeno disuelto fluctuó en ambos estratos, manteniendo el mismo patrón de distribución. En el Estrato 1 los valores máximos ocurrieron al inicio del periodo, variando de 6,2 a $8,6 \mathrm{mg} \mathrm{O} \cdot \mathrm{L}^{-1}$ y en el Estrato $2 \mathrm{de}$ 5,5 a $8,5 \mathrm{mg} \mathrm{O}_{2} \cdot \mathrm{L}^{-1}$ (Fig. 4b). Al comparar los valores entre ambos periodos (surgencia y no surgencia), la concentración de oxígeno en el Estrato 1 mostró diferencias significativas $(\mathrm{p} \leq 0,05)$, sugiriendo la influencia del viento sobre la superficie.

En periodo de no surgencia, la concentración de clorofila-a varió en el tiempo en ambos estratos, con el mismo patrón y los valores fueron ligeramente mayores en el Estrato 2 (Fig. 5a). Los menores valores se observaron al comienzo del estudio $(1,11$ $\mathrm{mg}$ Clor-a $\cdot \mathrm{m}^{-3}$ ) y se incrementaron ligeramente hasta llegar a un máximo al final del periodo $(8,57 \mathrm{mg}$ Clor$\left.\mathrm{a} \cdot \mathrm{m}^{-3}\right)$. En el periodo de surgencia la concentración de clorofila-a aumentó, con fluctuaciones respecto al primero $(\mathrm{p} \leq 0,05)$. En general, presentó el mismo patrón entre estratos y aumentó a mediados del tiempo en el Estrato 2, los valores fluctuaron de 4,21 a 44,59 $\mathrm{mg}$ Clor-a $\cdot \mathrm{m}^{-3}$ (Fig. 5b).

La concentración de la feofitina, que es indicativa del estado fisiológico de las algas, durante el periodo de no surgencia presentó el mismo patrón de variación, aumentando a finales del periodo $(\mathrm{p} \leq 0,05)$. En cambio en surgencia presentó fluctuaciones en ambos estratos, siendo mayor en el Estrato 2 ( $\mathrm{p} \leq$ $0,05)$ (Figs. 6a y 6b).

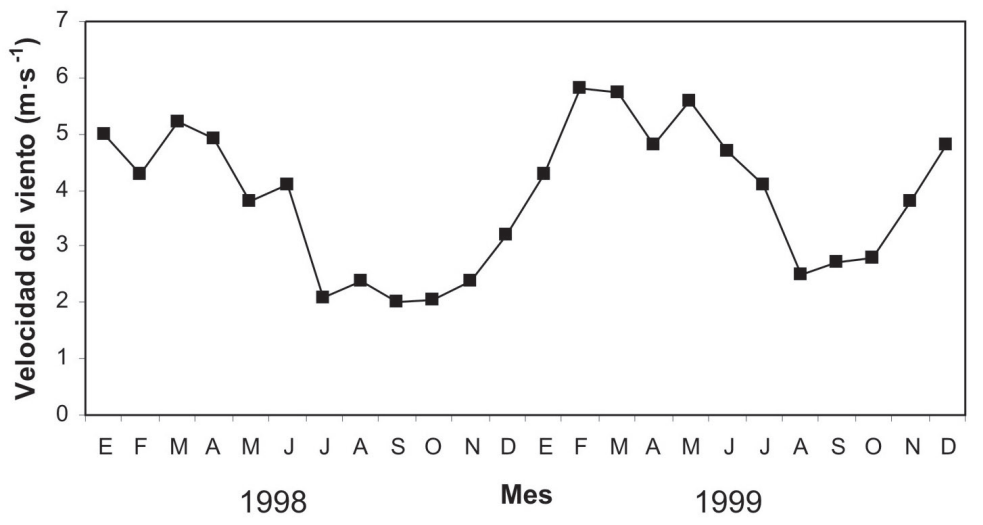

Figura 2. Variación de la velocidad del viento en 1998 y 1999.

Figure 2. Variation of wind speed during years 1998-1999.

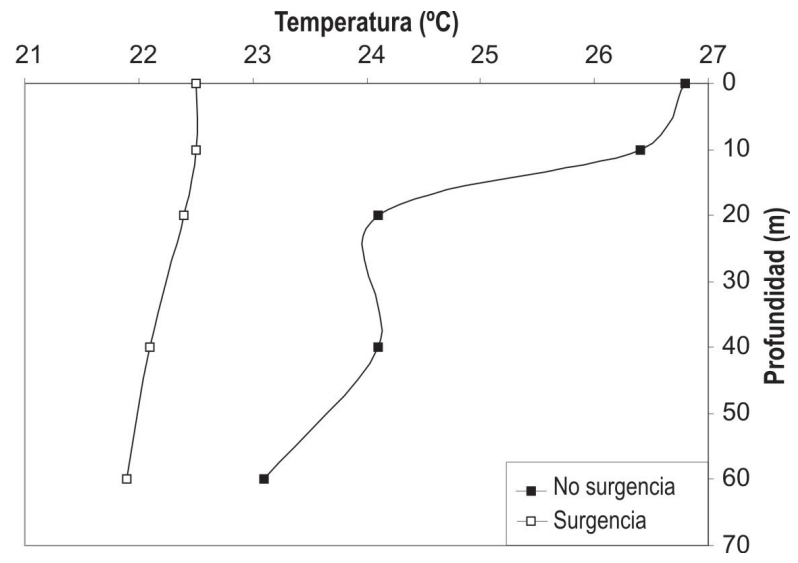

Figura 3. Variación vertical de la temperatura durante los periodos analizados (surgencia y no surgencia).

Figure 3. Vertical variation of temperature during both periods (upwelling and downwelling). 

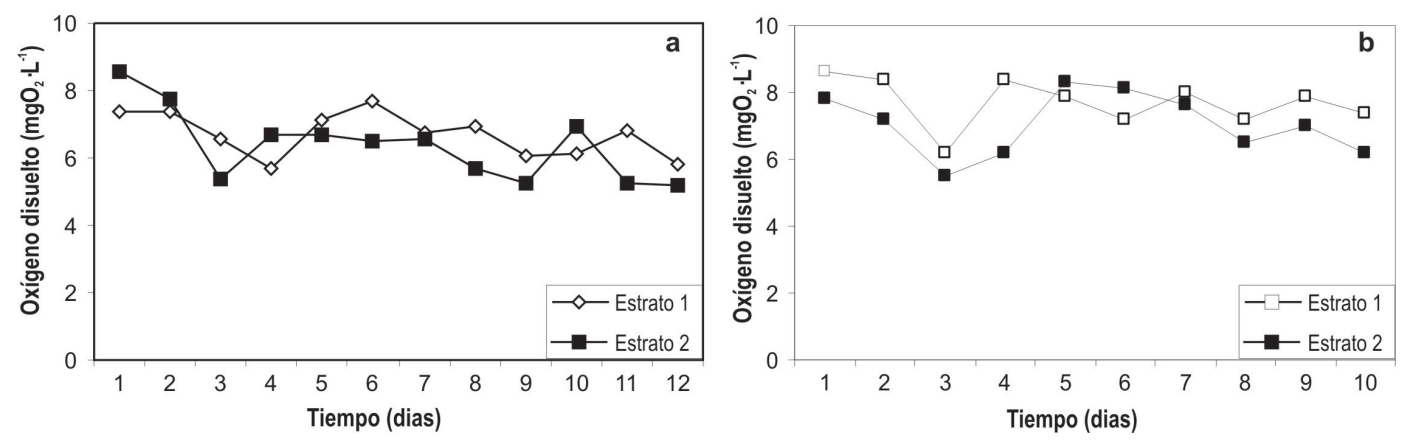

Figura 4. Variación diaria de la concentración de oxígeno disuelto en los periodos analizados. a) no surgencia, b) surgencia.

Figure 4. Daily variation of dissolved oxygen in both analyzed periods. a) downwelling, b) upwelling.
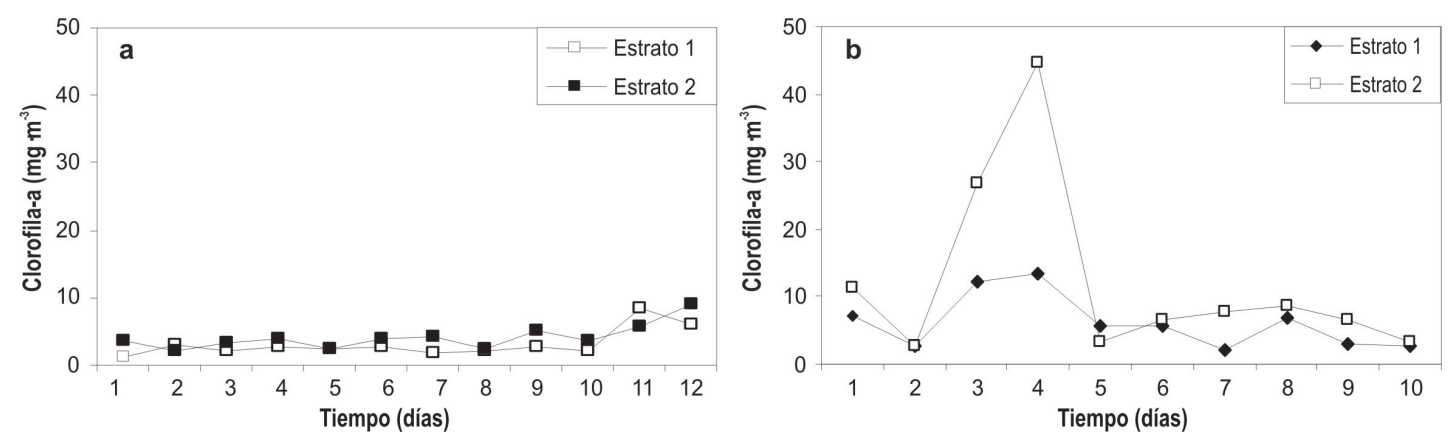

Figura 5. Variación diaria de la clorofila-a en los periodos analizados. a) no surgencia, b) surgencia.

Figure 5. Daily variation of chlorophyll-a in analyzed layers. a) downwelling, b) upwelling.
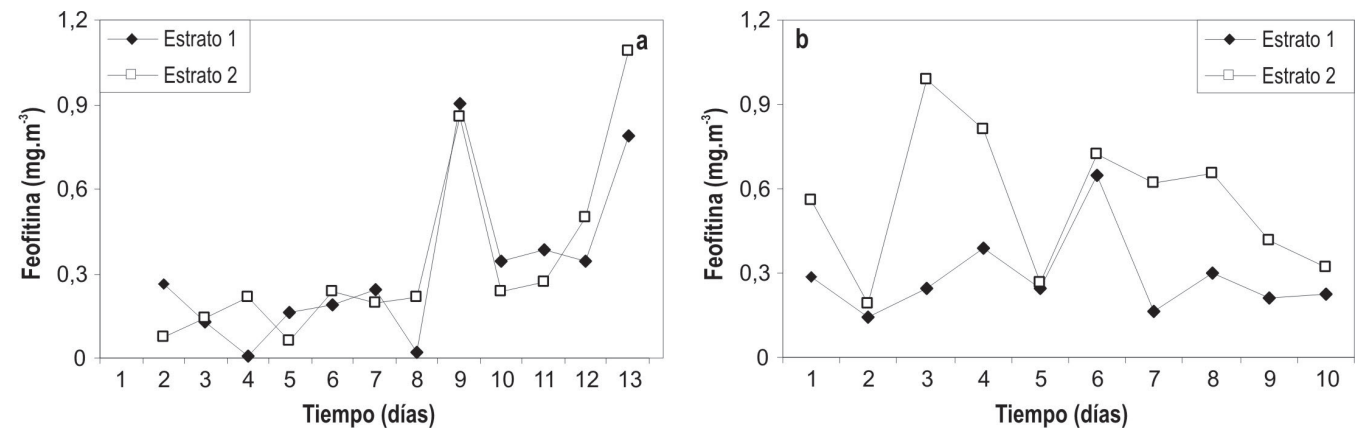

Figura 6. Variación diaria de la feofitina en los periodos analizados. a) no surgencia, b) surgencia.

Figure 6. Daily variation of phaeophytins in analyzed layers. a) downwelling, b) upwelling. 
La productividad primaria en el periodo de no surgencia, presentó en ambos estratos la misma fluctuación a lo largo del tiempo. Los valores fueron ligeramente altos al comienzo y superiores en el Estrato $2\left(0-417 \mathrm{mg} \mathrm{C} \cdot \mathrm{m}^{-3} \cdot \mathrm{h}^{-1}\right)$ (Figs. 7a y $\left.7 \mathrm{~b}\right)$. En cambio en el periodo de surgencia, presentó marcadas fluctuaciones en ambos estratos, con los mayores valores en el Estrato 1 (133-860 $\mathrm{mg} \mathrm{C} \cdot \mathrm{m}^{-}$ $\left.{ }^{3} \cdot \mathrm{h}^{-1}\right)$ (Fig. 8a), mientras que en el Estrato 2 presentó marcadas fluctuaciones en el tiempo, de 76 a $426 \mathrm{mg}$ $\mathrm{C} \cdot \mathrm{m}^{-3} \cdot \mathrm{h}^{-1}(\mathrm{p} \leq 0,05)$ (Fig. 8b). El análisis estadístico mostró que hubo diferencias significativas entre los periodos de no surgencia y surgencia $(p \leq 0,05)$. Es importante mencionar que la respiración en algunos puntos sobrepasó a la productividad bruta.

El Análisis de Componentes Principales con 38 casos completos incluyó las variables: productividad bruta, productividad neta, oxígeno disuelto y respiración. En los dos primeros componentes se obtuvo como resultado el $84,3 \%$ de la varianza total. Al ob-

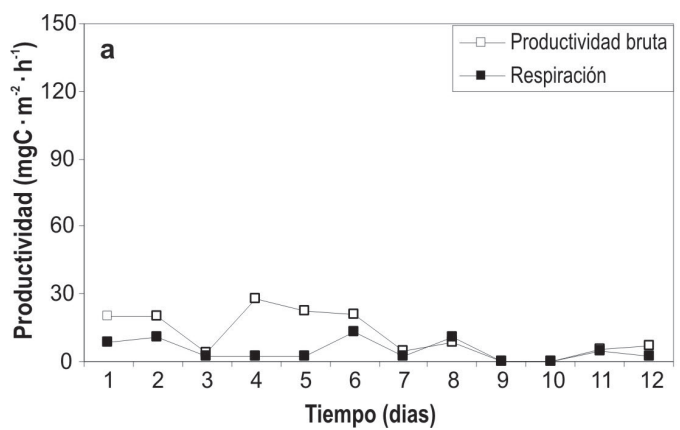

servar los pesos del Componente Principal se puede decir que la productividad neta no está relacionada con la productividad bruta y respiración, a pesar que estas tres tienen importancia similar (Fig. 9).

\section{DISCUSIÓN}

Según algunos autores (Kiorboe, 1993; Monteiro \& Largier, 1999), el ascenso de aguas subsuperficiales genera turbulencia en la columna de agua, modificando el ambiente físico y químico, aumenta la densidad de organismos y cambia la estructura vertical del plancton. La turbulencia implica mayor disponibilidad de nutrientes en la zona eufótica producto de la surgencia y altera la penetración de la luz por el crecimiento de fitoplancton. Este fenómeno causa cambios en la transparencia del agua y afecta la distribución vertical de fitoplancton que está determinada por la cantidad de energía solar y por el coeficiente de extinción de luz (Margalef, 1980).

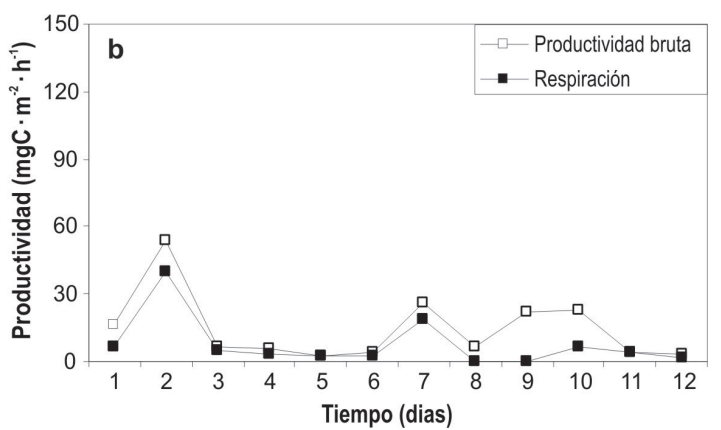

Figura 7. Variación diaria de la productividad bruta y respiración en el periodo de no surgencia. a) Estrato 1, b) Estrato 2.

Figure 7. Daily variation of gross productivity and breathing in dowmwelling period. a) Stratum 1, b) Stratum 2.
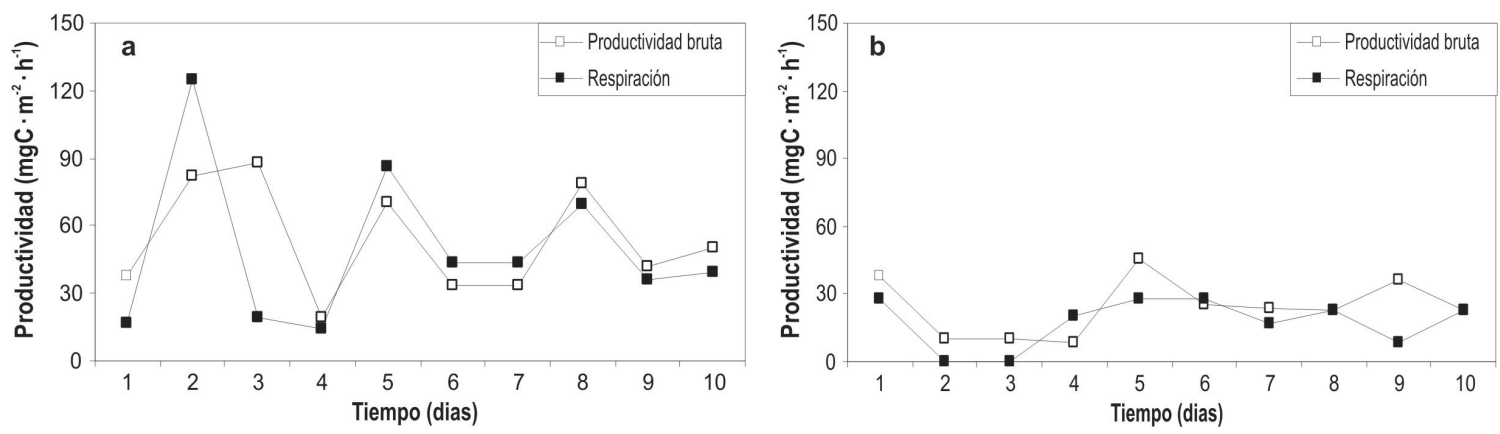

Figura 8. Variación diaria de la productividad bruta y respiración en el periodo de surgencia. a) Estrato 1, b) Estrato 2.

Figure 8. Daily variation of gross productivity and breathing in upwelling period. a) Stratum 1, b) Stratum 2. 


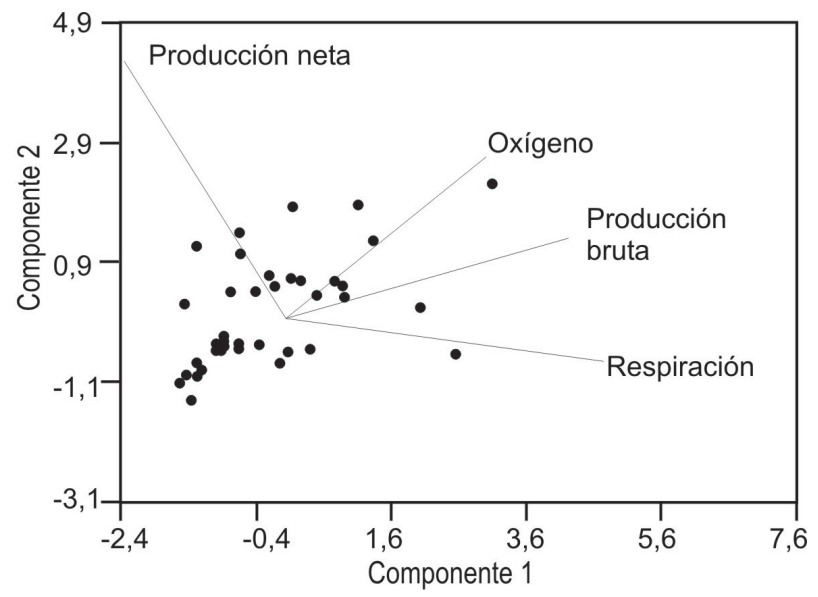

Figura 9. Biplot del Análisis de Componente Principal.

Figure 9. Biplot of the Principal Component Analysis.

La fluctuación vertical en la temperatura del agua, estratificada versus mezclada, correspondió con la mayor temperatura en superficie y la presencia de la termoclina. En cambio, en época de mezcla, se caracterizó por las fluctuaciones y persistencia de vientos locales. En la época de surgencia, la temperatura tuvo una pequeña diferencia $\left(0,6^{\circ} \mathrm{C}\right)$ entre la superficie y el fondo $(60 \mathrm{~m})$, que indica que la columna de agua estaba bien mezclada. En cambio en no surgencia, la diferencia fue de $3,35^{\circ} \mathrm{C}$ debido a la termoclina y a las variaciones diarias en la velocidad del viento. Esta diferencia en temperatura concuerda con lo expresado por diversos autores (Fukuoka, 1965; Okuda et al., 1968; Urosa, 1980; Bode et al., 1996), quienes señalan el ascenso de aguas más frías, dependiendo de la intensidad y duración de los vientos. Este proceso tiene alta importancia biológica porque cambia la columna de agua estratificada, de mayor temperatura y baja concentración de nutrientes, a valores bajos de temperatura y altas concentraciones de nutrientes.

Así también, se pudo observar que el pigmento predominante fue la clor-a y los valores mayores se observaron en el periodo de surgencia. Esto indica que en esta época, el fitoplancton en la bahía fue muy productivo y estaba en buenas condiciones fisiológicas, ya que su concentración respondió al enriquecimiento de nutrientes.

En general, se observó que entre las diferentes épocas, la concentración de clor-a mostró que la estructura vertical de los pigmentos concordó con los cambios en la composición y abundancia de las especies. Es decir, que la estructura de los pigmentos estuvo relacionada con cambios en la composición de especies, en la concentración de los pigmentos y en la fotoadaptación (Hoepffner \& Sathyendranath, 1992).

Cuando la concentración de feofitina con respecto a la clor-a fue alta en ambos periodos, tanto en la superficie como en el fondo, indica que en ese momento la calidad de los pigmentos fue menor o mayor el pastoreo del zooplancton. Esto coincide con Margalef (1980), ya que la feofitina es producto de la destrucción de los pigmentos del fitoplancton por alta luminosidad, pastoreo o senescencia, e indica el estado fisiológico de las microalgas.

La productividad primaria y respiración son dos procesos fundamentales que conducen el flujo de la materia orgánica en ecosistemas marinos y su relación es un índice ecológico importante, el cual define el estrato trófico en las comunidades biológicas. Según Moncoiffé Gwenaëlle et al. (2000) en los sistemas pelágicos con baja entrada de materia orgánica externa, la productividad primaria es el principal recurso de carbono orgánico y el balance entre los dos procesos, y define la cantidad de materia orgánica disponible en el sistema. Sin embargo, el grado de acoplamiento entre la producción y respiración de la comunidad está sujeto al número de interacciones físicas y biológicas responsables de ambos procesos en tiempo y espacio. La comparación de la productividad primaria calculada a diferentes escalas de tiempo, revela que tiene un efecto importante en la estimación del impacto de eventos de alta productividad en el ecosistema. Una vez estabilizada la columna de agua, se espera que haya una regeneración biológica vertical (Bode et al., 1996). 
Como se dijo anteriormente, en la época de surgencia, la incidencia de la luz solar fue menor. Esto influye en la productividad primaria, que tiene mayor relevancia en el estrato superficial, por la abundancia de fitoplancton, pero en el Estrato 2 disminuyó por la menor transparencia. Legendre \& Le Fèvre (1989), sugieren que el éxito de la productividad primaria, está influida por la variabilidad de procesos hidrodinámicos, tales como eventos de surgencia y periodos de transición temporal, ya que estos eventos proporcionan condiciones que promueven o inhiben la producción de organismos de diferente talla.

La tasa de productividad primaria estimada está dentro de los rangos señalados para otras áreas de surgencia, como Benguela $\left(40-340 \mathrm{mg} \mathrm{C} \cdot \mathrm{m}^{-2} \cdot \mathrm{d}^{-1}\right)$ (Gibbons et al., 1999) y Perú (1.000 $\left.\mathrm{mg} \mathrm{C} \cdot \mathrm{m}^{-2} \cdot \mathrm{d}^{-1}\right)$ (Bode et al., 1996). El ciclo de la surgencia en la bahía de Mochima, en general, mostró un patrón similar al de otras áreas de surgencia descrito por varios autores (Legendre, 1990; Bode et al., 1996; Tang-Dang-Ling et al., 1999; Schumann, 1999), desde el punto de vista de cambios en el patrón de temperatura, transparencia, abundancia de fitoplancton, productividad primaria y abundancia de zooplancton.

La escasa relación que hay con la productividad neta se debería a los detritos, aumento de la respiración dentro de las botellas BOD y al pastoreo de los herbívoros. Durante ambos periodos, los diferentes eslabones del plancton presentaron fuertes cambios temporales intradiarios, que se manifestaron de acuerdo a los denominados eventos Lasker.

\section{REFERENCIAS}

American Public Health Association (APHA). 1975. Standard methods for the examination of waters and waste water. APHA, Washington D.C., 1193 pp.

Bakun, A. 1973. Coastal upwelling indices, west coast of North America 1946-71. U.S. Departmenrt of Commerce, NOAA Technical Report, NHFSSSRS-693, 113 pp.

Bode, A., B. Casas, E. Fernandez, E. Maranon, P. Serret \& M. Varela. 1996. Phytoplankton biomass and production in shelf waters off NW Spain: spatial and seasonal variability in relation to upwelling. Hydrobiologia, 341(3): 225-234.

Cury, P. \& C. Roy. 1989. Optimal environment window and pelagic fish recruitment success in upwelling areas. Can. J. Fish. Aquat. Sci., 46: 670-680.
Chatfeld, C. \& A.J. Collins. 1980. Introduction to multivariate analysis. Chapman \& Hall, New York, $246 \mathrm{pp}$.

D'Croz, L., J. del Rosario \& A. Gómez. 1991. Upwelling and phytoplankton in the bay of Panamá. Rev. Biol. Trop., 39(2): 233-241.

Dickey, T. 1990. Physical-optical-biological scales relevant to recruitment in large marine ecosystems. En: K. Sherman, M. Lewis \& B. Gold (eds.). Large patterns marine processes ecosystems and yields. Am. Assoc. Adv. Sci., Washington D.C., 242 pp.

Fortier, L. \& J. Gagné. 1990. Larvae herring (Clupea harengus) dispersion, growth and survival in the St. Lawrence estuary: Match/ Mismath or membership / Vagrancy? Can. J. Fish. Aquat. Sci., 47: 1898-1912.

Fukuoka, J. 1965. Coastal upwelling near Venezuela. 1. Year to year changes of upwelling. Bol. Inst. Oceanogr. UDO, 4: 223-233.

Fukuoka, J. 1966. Coastal upwelling near Venezuela. 2. Certain periodicities of hidrographic conditions. Bol. Inst. Oceanogr. UDO, 5: 84-95.

Gibbons, M.J., N. Gugushe, A.J. Boyd, L.J. Shannon \& B.A. Mitchell-Innes. 1999. Changes in the composition of the non-copepod zooplancton assemblage in St Helena bay (southern Benguela ecosystem) during a six day drogue study. Mar. Ecol. Prog. Ser., 180: 111-120.

Gómez Gaspar, A. 1996. Causas de la fertilidad marina en el nororiente de Venezuela. Interciencia, 21(3): 140-146.

Hoepffner, N. \& L. Sathyendranath. 1992. Bio-optical characteristics of coastal waters: Absorption spectra of phytoplankton and pigment distribution in the western North Atlantic. Limnol. Oceanogr., 37(8): 1660-1679.

Kato, K. 1961. Some aspects on biochemical characteristics of sea water and sediments in Mochima bay, Venezuela. Bol. Inst. Oceanogr. UDO, 1(2): 343-350.

Kiorboe, T. 1993. Turbulence, phytoplankton cell size, and the structure of pelagic food webs. Adv. Mar. Biol., 29: 72 pp.

Lasker, R. 1975. Field criteria for survival of anchovy larvae: The relation between inshore chlorophyll maximum layers and successful first feeding. US. Fish. Bull., 73(3): 453-462. 
Legendre, L. \& J. Le Fèvre. 1989. Hydrodynamical singularities as controls of recycled versus export production in oceans. En: W.H. Berger, V.S. Smetacek \& G. Wefer (eds.). Productivity of the ocean: present and past. John Wiley \& Sons, New York, $163 \mathrm{pp}$.

Legendre, L. \& J. Le Fèvre. 1990. The significance of microalgal blooms for fisheries and for export of particulate organic carbon in oceans. J. Plankton Res., 12: 681-699.

Marín, B. 1996. Transport et recrutement du muge argenté, Mugil curema dans une lagune côtière tropicale. Tesis Doctoral, Université Laval Quebec, Canadá, 104 pp.

Margalef, R. 1965. Ecological correlations and the relationships primary production and comunity structure. Mem. Inst. Ital. Idrobiol., 18(suppl): 355365.

Margalef, R. 1980. Ecology. Editorial Omega. Barcelona, 95 pp.

Moncoiffe Gwenaëlle, X., A. Alvares-Salgado, F.G. Figeiras \& G. Savidge. 2000. Seasonal and shorttime-scale dynamics of microplankton community production and respiration in an inshore upwelling system. Mar. Ecol. Prog. Ser., 196: 111-126.

Montecinos, A. \& F. Balbontín. 1993. Indices de surgencia y circulación superficial del mar: implicaciones biológicas en un área de desove de peces entre Los Vilos y Valparaíso, Chile. Rev. Biol. Mar. Valparaíso, 28(1): 133-150.

Monteiro, P.M. \& J.L. Largier. 1999. Thermal stratification in Saldanha Bay (South Africa) and subtidal, density-driven exchange with the coastal waters of the Benguela Upwelling System. Estuar. Coast. Shelf Sci., 49(6): 877-890.
Mullin, M. 1993. Webs \& scales: physical and ecological processes in marine fish recruitment. Books in recruitment fishery oceanography. Washington Sea Grant Program. Distribute by The University of Washington Press, Seattle and London, $135 \mathrm{pp}$.

Okuda, T., J. Benítez, A.J. García \& E. Fernández. 1968. Condiciones hidrográficas y químicas de la Bahía de Mochima y la Laguna Grande del Obispo desde 1964 a 1966. Bol. Inst. Oceanogr. UDO, 7: 7-37.

Osborn, T., H. Yamasaki \& K. Squires. 1990. Direct simulation of the effect of turbulence on planktonic contact rates. En: K. Sherman, M. Lewis \& B. Gold (eds.). Large patterns marine processes ecosystems and yields. Am. Assoc. Adv. Sc., 242 pp.

Schumann, E.H. 1999. Wind-driven mixed layer and coastal upwelling processes off the south coast of South Africa. J. Mar. Res., 57(4): 671-691.

Strickland, J.D. 1960. Measuring the production of marine phytoplancton. Bull. Fish. Res. Bd. Can., 167-172.

Strickland, J.D. \& T.R. Parsons. 1968. A practical handbook of sea water analysis. Bull. Fish. Res. Bd. Can., 167: 131 pp.

Tang, Dang Ling, Ni. L. Hsun, D.R. Kester, \& F.E Müeller Karger. 1999. Remote sensing observations of winter phytoplankton blooms southwest of the Luzon Strait in the South China Sea. Mar. Ecol. Prog, Ser., 191: 43-51.

Urosa, L. 1980. Nauplii balanomorfos y variación diurna de la temperatura en la ensenada de Turpialito, Venezuela. Bol. Inst. Oceanogr. UDO, 19(1-2): 73-91.

Velilla, S. 1995. Diagnostics and robust estimation in multivariate analysis transformations. J. Amer. Stat. Ass., 90: 945-951. 
